\title{
BMJ
}

\section{ExStroke Pilot Trial of the effect of repeated instructions to improve physical activity after ischaemic stroke: a multinational randomised controlled clinical trial}

\author{
Gudrun Boysen, professor of neurology, ${ }^{1}$ Lars-Henrik Krarup, PhD student, ${ }^{1,6}$ Xianrong Zeng, neurologist, ${ }^{2}$ \\ Adam Oskedra, neurologist, ${ }^{3}$ Janika Kõrv, neurologist, ${ }^{4}$ Grethe Andersen, neurologist, ${ }^{5}$ Christian Gluud, head \\ of Copenhagen Trial Unit, ${ }^{6}$ Anders Pedersen, study nurse, ${ }^{1}$ Marianne Lindahl, physiotherapist, ${ }^{1}$ Lotte Hansen, \\ physiotherapist, ${ }^{1}$ Per Winkel, statistical adviser, ${ }^{6}$ Thomas Truelsen, neurologist ${ }^{1}$ for the ExStroke Pilot Trial \\ Group
}

${ }^{1}$ Department of Neurology, Bispebjerg Hospital, Copenhagen University Hospital, Bispebjerg

Bakke 23, DK-2400

Copenhagen NV, Denmark

${ }^{2}$ Department of Neurology, Sichuan Provincial People's Hospital, Chengdu, China

${ }^{3}$ Department of Neurology, Institute of Psychiatry and Neurology, Warsaw, Poland ${ }^{4}$ Department of Neurology and Neurosurgery, University of Tartu, Estonia

${ }^{5}$ Department of Neurology, Aarhus University Hospital, Denmark

${ }^{6}$ Copenhagen Trial Unit, Centre for Clinical Intervention Research, Rigshospitalet, Copenhagen University Hospital

Correspondence to: $\mathrm{G}$ Boysen gb01@bbh.regionh.dk

Cite this as: BMJ 2009;339:b2810 doi:10.1136/bmi.b2810

\section{ABSTRACT}

Objectives To investigate if repeated verbal instructions about physical activity to patients with ischaemic stroke could increase long term physical activity.

Design Multicentre, multinational, randomised clinical trial with masked outcome assessment.

Setting Stroke units in Denmark, China, Poland, and Estonia.

Participants 314 patients with ischaemic stroke aged $\geq$ 40 years who were able to walk-157 (mean age 69 .

7 years) randomised to the intervention, 157 (mean age 69.4 years) in the control group.

Interventions Patients randomised to the intervention were instructed in a detailed training programme before discharge and at five follow-up visits during 24 months. Control patients had follow-up visits with the same frequency but without instructions in physical activity. Main outcome measures Physical activity assessed with the Physical Activity Scale for the Elderly (PASE) at each visit. Secondary outcomes were clinical events.

Results The estimated mean PASE scores were 69.1 in the intervention group and 64.0 in the control group (difference 5.0 (95\% confidence interval -5.8 to 15.9 ), $\mathrm{P}=0.36$. The intervention had no significant effect on mortality, recurrent stroke, myocardial infarction, or falls and fractures.

Conclusion Repeated encouragement and verbal instruction in being physically active did not lead to a significant increase in physical activity measured by the PASE score. More intensive strategies seem to be needed to promote physical activity after ischaemic stroke.

Trial registration Clinical Trials NCT00132483

\section{INTRODUCTION}

In observational studies even a moderate level of physical activity has been associated with a reduction of the risk of cardiovascular disease and of first stroke. ${ }^{1-7}$ The influence of physical activity on the risk of recurrent stroke, however, is unknown. Nevertheless, physical exercise is often recommended for stroke survivors because it is assumed that physical activity may favourably influence the prognosis through its effect on blood pressure, glucose metabolism, and cholesterol level. ${ }^{8}$ Counselling on physical activity had shown some effect in elderly sedentary people, ${ }^{9}$ and in patients with diabetes, hypertension, hypercholesterolaemia, and overweight. ${ }^{11}$ However, authors of systematic reviews of interventions to promote physical activity found the evidence insufficient to assess its effectiveness. ${ }^{1213}$ A number of small randomised trials have shown that supervised physical training can improve stroke patients' balance, walking ability, and physical fitness. ${ }^{14-18}$ These trials usually lasted three to six months.

The ExStroke Pilot Trial was designed to assess if repeated encouragement and verbal instructions regarding how to exercise could result in a sustained increase in stroke patients' physical activity as measured by the physical activity scale for the elderly (PASE). ${ }^{1920}$ The rationale and design of the ExStroke Pilot Trial have been described previously. ${ }^{21}$

\section{METHODS}

Trial participants

Patients with ischaemic stroke aged $\geq 40$ years from six stroke units in Denmark and from one neurological department each in Chengdu, China, Warsaw, Poland, and Tartu, Estonia, were eligible if they were able to walk unassisted. Canes and walkers were allowed. Participants were enrolled within 90 days of onset of stroke symptoms. Verbal and written informed consent were obtained prior to enrollment. Exclusion criteria were inability to understand the meaning of the trial, unwillingness to participate, medical contraindications to exercise, or a modified Rankin scale of 4 or 5 before the qualifying event. The modified Rankin scale for activities of daily living ranges from 0 to 5 , where 0 means no limitations and 5 means confinement to bed and requiring constant help. ${ }^{2223}$ Stroke severity 
was assessed at baseline with the Scandinavian stroke scale (SSS), which ranges from 0 to 58 , the latter meaning no deficits in the measured items. ${ }^{24}$

Patient enrolment started in August 2003 and was completed in October 2005. In the Danish department that included most participants only about $15 \%$ of stroke patients were recruited into the trial. ${ }^{21}$ About $15 \%$ expressed unwillingness to participate because of reluctance to engage in physical training. Other reasons for non-eligibility were fatal stroke, severe neurological deficits, dysphasia or cognitive impairment, discharge to nursing homes, transfer to other departments due to comorbidity, enrolment in other trials, or age below 40 years.

\section{Interventions}

The experimental intervention consisted of repeated encouragement and verbal instruction on being physically active given by a physiotherapist, except for the Chinese centre, where a neurologist provided the instruction. The control group received information on the possible benefits of physical activity but no specific instruction. Both groups received standard treatment with antithrombotic drugs, antihypertensive treatment, and statins as needed after individual assessment. Physiotherapists and physicians examined patients

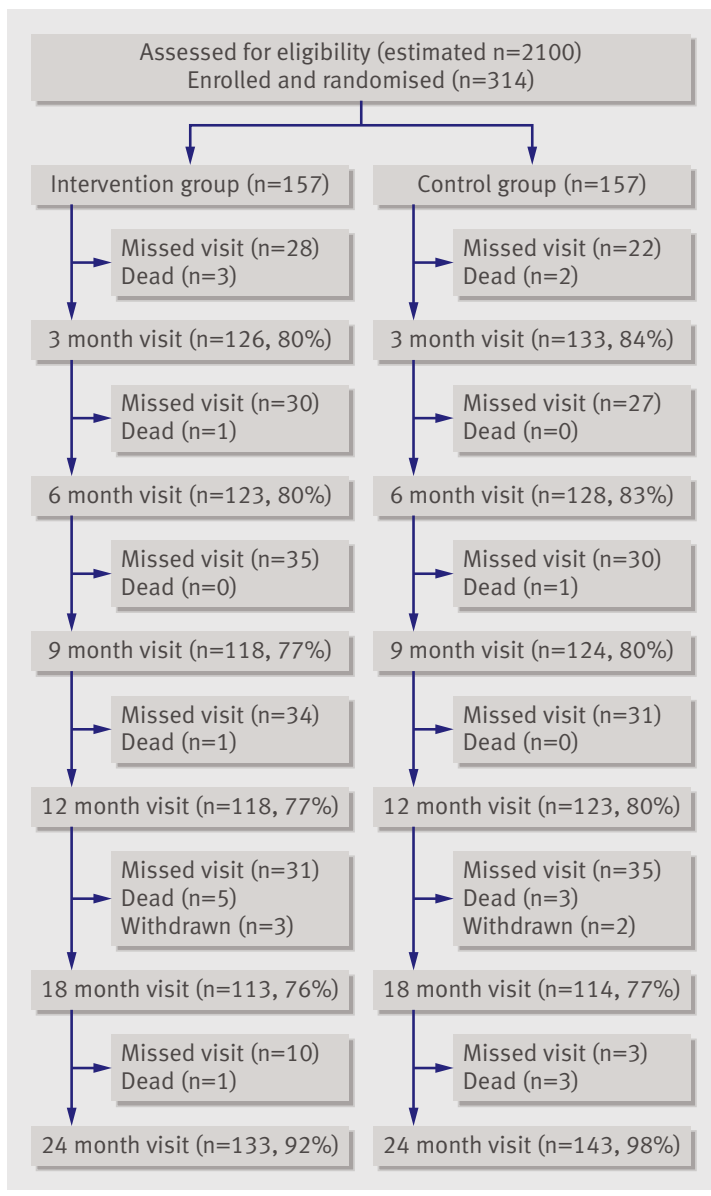

Fig 1| Participant flow chart of ExStroke Pilot Trial. Withdrawn denotes patients withdrawn due to severe recurrent stroke. during their hospital stay, and rehabilitation was carried out according to the customs of the department.

Patients randomised to the intervention group met up with a trial physiotherapist (or neurologist in China) for instruction in a detailed training programme to start after discharge from the hospital. At the first session the instructor spent about 30-60 minutes getting acquainted with the participant and evaluating the consequences of the stroke. Considerable effort was taken to motivate the participant. The aim was to make the participant choose the most suitable types of physical activity for him or her. The programme was individualised according to each patient's resources, former activities, and preferences. Patients were encouraged to use facilities for physical activity in their local community. They were encouraged to use fitness centres, to walk several kilometres a day (possibly as Nordic walking with sticks to improve balance), to go to public swimming pools, and to exercise in their local senior centre. Bicycling (with a cycle helmet) was also encouraged for those who cycled. At each visit the instructor and participant would fill in a standard agreement form with various choices of physical activity (see extra material on bmj.com), with one copy of the completed form for the participant to take home and another to be kept in the participant's file.

At the follow-up visits - every three months during the first year, and thereafter every six months until the end of the trial-the instructor spent about 20-30 minutes with the participant on repeated instructions and readjustment of the physical activity plan. Between visits a telephone call was made to remind each participant in the intervention group about the physical activity agreement. Participants were asked in detail about their activities and encouraged to increase efforts and to exercise more, and were told that to become sweaty and short of breath was desirable.

No telephone calls were made in the control group. Participants in the control group received standard treatment without detailed information on physical activity. They were seen for clinical visits with the same frequency as the intervention group.

\section{Assessment of physical activity}

At their entry to the study, we ascertained participants' level of physical activity during the week before their stroke, and at each follow-up visit we ascertained their level of physical activity in the week preceding the visit. The physical activity scale for the elderly (PASE) ${ }^{1920}$ has 12 questions about different activities (walking outside the home, light sport, moderate sport, strenuous sport, exercises to increase muscle strength and endurance, light housework, heavy housework, home repairs, lawn work or yard care, caring for another person, work for pay, and work as a volunteer), and each question has subsidiary questions as to the frequency per week and the time per day spent on the activity. The PASE score can range from 0 to over 400, with a higher score meaning a higher level of physical activity. The validity and reliability of the PASE questionnaire has been tested in several 
Table 1|Entry characteristics for all patients in the ExStroke Pilot Trial. Values are numbers (percentages) of patients unless stated otherwise

Intervention group $(\mathrm{n}=157) \quad$ Control group $(\mathrm{n}=157)$

Median (IQR) age (years)

$69.7(60.0-77.7)$ $69.4(59.6-75.8)$

Median (IQR) PASE score before stroke

$76(50-124)$

Women

Median (IQR) Scandinavian stroke scale

68 (43.3)

$54(51-58)$

Mean (SD) body mass index

$25.8(4.2)$

$152.7(21.1)$

$65(50-106)$

Mean (SD) systolic blood pressure $(\mathrm{mm} \mathrm{Hg})$

$22(14.0)$

Stroke
Transient ischaemic attack

Atrial fibrillation

Myocardial infarction

Diabetes

Intermittent claudication

Depression

14 (8.9)

25 (15.9)

$13(8.3)$

$28(17.8)$

$15(9.6)$

$21(13.4)$

$93(59.2)$

Coronary artery bypass grafting

$5(3.2)$

Percutaneous transluminal coronary angioplasty

Blood:

\begin{tabular}{|c|c|c|}
\hline Mean (SD) total cholesterol (mmol/l) & $5.3(1.2)$ & $5.3(1.3)$ \\
\hline Median (IQR) glucose (mmol/l) & $5.6(5.1-6.4)$ & $5.6(5.1-6.3)$ \\
\hline Median (IQR) C reactive protein $(\mathrm{mg} / \mathrm{l})$ & $5.0(2.4-12.0)$ & $6.0(3.0-11)$ \\
\hline \multicolumn{3}{|l|}{ Modified Rankin score: } \\
\hline 0 & $114(72.6)$ & $116(73.9)$ \\
\hline 1 & $28(17.8)$ & $20(12.7)$ \\
\hline 2 & $13(8.3)$ & $18(11.5)$ \\
\hline 3 & $2(1.3)$ & $3(1.9)$ \\
\hline \multicolumn{3}{|l|}{ Education (years): } \\
\hline$\leq 8$ & $71(45.2)$ & $73(46.5)$ \\
\hline $9-12$ & $53(33.8)$ & $63(40.1)$ \\
\hline$\geq 13$ & $33(21.0)$ & $21(13.4)$ \\
\hline \multicolumn{3}{|l|}{ Smoking status: } \\
\hline Current & $49(31.2)$ & $66(42.0)$ \\
\hline Former & $62(39.5)$ & $46(29.3)$ \\
\hline Never & $46(29.3)$ & $45(28.7)$ \\
\hline \multicolumn{3}{|l|}{ Alcohol intake (units): } \\
\hline 0 & $32(20.4)$ & $46(29.5)$ \\
\hline «14(women)/21(men) & $108(68.8)$ & $89(57.1)$ \\
\hline >14(women)/21(men) & $17(10.8)$ & $21(13.4)$ \\
\hline \multicolumn{3}{|l|}{ Stroke diagnosis: } \\
\hline Large artery disease & $40(25.5)$ & $29(18.5)$ \\
\hline Cardioembolism & $21(13.4)$ & $19(12.1)$ \\
\hline Small vessel disease & $51(32.5)$ & $51(32.5)$ \\
\hline Other determined cause & 0 & $2(1.3)$ \\
\hline Undetermined cause & $45(28.7)$ & $56(35.7)$ \\
\hline
\end{tabular}

$\mathrm{IQR}=$ interquartile range; $\mathrm{SD}=$ standard deviation; PASE = physical activity scale for the elderly.

studies, ${ }^{192025-29}$ and it is reliable for interview, mail, and telephone administration ${ }^{2728}$ and valid in a variety of population groups, including those with disability. The PASE score was positively and significantly correlated $(r=0.68(95 \%$ confidence interval 0.35 to 0.86$))$ with the doubly labelled water method, which is considered the gold standard for measuring energy expenditure. ${ }^{25}$ In a random sample of 49 participants in the ExStroke Pilot Trial we tested if their PASE score reflected their physical capacity. ${ }^{29} \mathrm{We}$ found a significant correlation between PASE score and their score on the senior fitness test, ${ }^{30}$ which reflects the capacity to perform everyday activities during functional examination of strength, aerobic capacity, and balance.

\section{Study objectives}

Our objective was to assess if repeated verbal encouragement and instruction in being physically active would result in a long term improvement in the level of physical activity, and, further, to explore the effect of the intervention on mortality and new cerebrovascular and cardiovascular events, on falls and fractures, and on the modified Rankin scale. ${ }^{2223}$

\section{Outcome measures}

The primary outcome was the difference in PASE score between the two groups. Secondary outcomes included the time from randomisation to recurrent stroke, myocardial infarction, or death (from any cause). Other outcomes were time to vascular death, frequency of recurrent stroke, modified Rankin scale, and falls and fractures.

Stroke was defined as the sudden onset of a neurological deficit with symptoms continuing for more than 24 hours or leading to death with no apparent cause other than vascular. In all such patients the results of computed tomography or magnetic resonance imaging were compatible with a stroke diagnosis. Diagnosis of myocardial infarction followed international guidelines. Vascular death was defined as death from stroke, myocardial infarction, or other vascular causes or sudden unexpected death. Falls were defined as sudden unintentional contact with the floor or ground. All events were adjudicated by an independent adjudication committee, which was blinded to the intervention group of the patient.

\section{Sample size}

The sample size calculation assumed a minimal relevant mean difference of 20 PASE points between the intervention and the control groups and a standard deviation of 50 PASE points. Based on the sample size calculation $(\alpha=0.05, \beta=0.20), 99$ patients would be needed in each group. ${ }^{21} \mathrm{~A}$ total of 300 patients were planned to be included to allow for dropouts. A difference of 20 PASE points corresponds to an increase of physical activity (such as walking outside the home for two hours three times a week) that was considered a realistic goal and which is associated with an odds ratio of 0.72 (95\% confidence interval 0.59 to 0.89 ) of first stroke. ${ }^{28}$

\section{Randomisation}

The ExStroke Pilot Trial is a randomised, multicentre, multinational clinical trial with masked outcome assessment in patients with ischaemic stroke. The participants were centrally randomised to intervention group or control group. Generation of allocation sequences was computer based. Allocation 

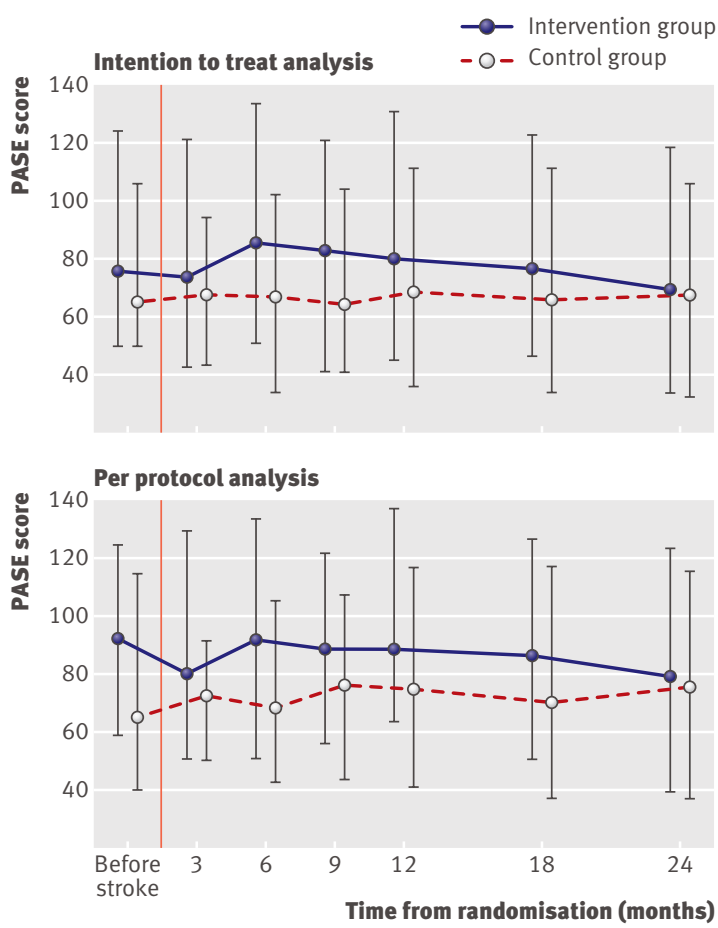

Fig 2 Participants' median (interquartile range) scores on the physical activity scale for the elderly (PASE) at each follow-up visit. Data are shown for the intention to treat analysis (top) and the per protocol analysis for participants who attended all planned visits (bottom)

concealment was achieved through centralised randomisation by telephone or email. The randomisation was stratified with regard to sex, age, stroke severity, and centre. Stratification was conducted into two groups for age (40-70 years, $\geq 70$ years), two groups for stroke severity (Scandinavian stroke scale 20-39 points, 40-58 points), and two groups for centre (Danish, or non-Danish) in blocks of 10, randomising patients 1:1 to experimental and control intervention. Block size was unknown to investigators.

\section{Randomisation implementation}

The investigator, who was informed from the Copenhagen Trial Unit via telephone or email whether a patient was randomised to intervention or control group, told the patient the result of the randomisation. For those in the intervention group the investigator arranged an appointment with the physiotherapist or provided the intervention herself.

\section{Masking and blinding}

In each centre an interviewer masked to the randomisation of the patient obtained the PASE score at the follow-up visits. These interviewers were physicians, medical students, or secretaries who were otherwise uninvolved in the conduct of the trial. They were instructed in how to use the PASE questionnaire and were told not to ask the patients about the group assignment. These investigators also obtained information about recurrent stroke, myocardial infarction, and falls - events that were adjudicated by a blinded adjudication committee.

\section{Statistical methods}

The data were analysed on the basis of intention to treat and of per protocol. A mixed model analysis (proc mixed SAS 9.1) including the repeated measures option was used to assess the time course of PASE. It proved necessary to code the data by using a square root transformation of the PASE scale (the variability of the PASE score increased with increasing score, and the square root scale eliminated this variability and improved normality). To obtain an estimate of the effect of the intervention from the mixed model program, the mean square root PASE in each group was calculated. By squaring the two mean values and calculating their difference, we obtained the difference between the two groups in the original scale. The standard error of the difference in mean PASE score was then calculated by using the fact that the standard error of the difference between the two mean values comprises (almost exactly) the same percentage of this difference whether the original scale or the square root scale is used.

The per protocol analysis included the patients who attended all six follow-up visits. To compensate for differences in pre-stroke PASE score between the two groups, we included the square root transformed prestroke PASE score as a covariate.

The difference in modified Rankin scale between the two groups at 3, 6, 12, and 24 months after randomisation was assessed with a non-parametric test (MannWhitney). Cox analyses were used to assess the effect of the intervention on time to a clinical event.

\section{Registration of the trial}

The protocol was approved by local ethics committees in Denmark (KF11006/04) and in the countries of the participating centres. The trial was registered with the Danish Data Protection Agency (J No 2003-41-3564) and at www.ClinicalTrials.gov (NCT00132483).

\section{Independent safety and monitoring board}

The Independent Safety and Monitoring Board conducted one interim analysis. There was no safety concern as to continuing the trial.

\section{RESULTS}

Figure 1 shows the participant flow in the trial. The estimated number of individuals assessed for eligibility is based on data from one department in which $15 \%$ of patients admitted for cerebrovascular disease were enrolled in the trial. A total of 314 patients fulfilled the entry criteria, agreed to participate, and were randomised between August 2003 and October 2005.

\section{Protocol deviations}

For one participant randomised to the intervention group, the PASE score before stroke was not obtained. Two patients randomised to the intervention group 
Table 2 Participants' median scores on the physical activity scale for the elderly (PASE) and number attending the follow-up visits in the intention to treat analysis of the ExStroke Pilot Trial

\begin{tabular}{|c|c|c|c|c|}
\hline \multirow[b]{2}{*}{ Visit } & \multicolumn{2}{|c|}{ Intervention group } & \multicolumn{2}{|c|}{ Control group } \\
\hline & Median (IQR) & No (\%) & Median (IQR) & No (\%) \\
\hline Before stroke & $76(50-124)$ & $156(99)$ & $65(50-126)$ & $157(100)$ \\
\hline \multicolumn{5}{|l|}{ Follow-up visit: } \\
\hline 3 month & $73(42-120)$ & $126(80)$ & $68(43-94)$ & $133(84)$ \\
\hline 6 month & $86(50-133)$ & $123(80)$ & $67(33-102)$ & $128(83)$ \\
\hline 9 month & $83(41-120)$ & $118(77)$ & $64(41-104)$ & $124(80)$ \\
\hline 12 month & $80(45-130)$ & $118(77)$ & $69(36-111)$ & $123(80)$ \\
\hline 18 month & $76(46-123)$ & $113(74)$ & $66(33-111)$ & $114(74)$ \\
\hline 24 month & $69(33-118)$ & $133(91)$ & $68(32-106)$ & $143(97)$ \\
\hline
\end{tabular}

$\mathrm{IQR}=$ interquartile range; $\%=$ percentage of survivors.

were not instructed in physical activity: they were followed up as if belonging to the control group but analysed according to randomisation. Two other participants did not get instruction in physical activity because of intercurrent illness. Participants who sustained a recurrent stroke continued in the trial if possible or (in the case of three participants in the intervention group and two in the control group) were withdrawn if neurological deficits were severe.

\section{Baseline demographics}

Table 1 shows the characteristics of the patients at entry to the study. The two groups were similar in terms of sex, age, and stroke severity (a median of 54 points on the Scandinavian stroke scale in both groups, corresponding to mild stroke). Pre-stroke PASE scores tended to be higher in the intervention group (median 76 (interquartile range 50-124)) than in the control group (65 (50-106)). Distribution of atrial fibrillation, diabetes, mean systolic arterial blood pressure, blood glucose, hypercholesterolaemia, pre-stroke modified Rankin scale, and habits of smoking and alcohol consumption were similar in the two groups.

The number of participants at each follow-up visit is given in fig 1 and table 2. For participants who were unable to visit the clinic at 24 months, a telephone interview was made-for 12 participants in the intervention group and 14 in the control group. Ten participants in the intervention group and two in the control group could not be reached and were considered lost to follow-up, but they were known to be alive and not having been hospitalised for stroke or myocardial infarction. In the intervention group, 80 participants had all six of the planned intervention sessions, 22 had five intervention sessions, 19 had four, 11 had three, 12 had two, nine had one, and four had none.

\section{Primary outcome measure}

The mean PASE scores in the intervention group versus the control group, estimated as stated above, were $69.1 v 64.0$, respectively (difference 5.0 (95\% confidence interval -5.8 to 15.9$), \mathrm{P}=0.36$ ).

Figure 2 shows the median PASE scores with interquartile range in the two groups. In the intention to treat analysis (top panel), the change in score over time was not significantly different between the groups, although the intervention group at 6 and 9 months' follow-up showed a non-significant increase in PASE score. The control group maintained the pre-stroke PASE score throughout the trial. Table 2 shows that the small difference between groups in PASE score that was apparent during the week preceding the stroke was maintained during most of the trial period but vanished at 24 months. There was no significant effect of centre and no significant interactions between protocol specified variables and the intervention indicator. The per protocol analysis (lower panel fig 2) of the patients who attended all planned follow-up visits (80 patients in the intervention group and 81 patients in the control group) showed a significant $(\mathrm{P}=0.03)$ difference in prestroke PASE score. Overall, there was no significant difference between the two groups regarding PASE score when adjusted for pre-stroke PASE score.

Of the points that constituted the PASE score, 15\% originated from walking outside the home, $46 \%$ from household activities, and 13\% from yard work. Sports activities accounted for only $10 \%$, caring for another person for $7 \%$, and work for $8 \%$. There were no significant differences between the groups in the distribution of activities.

\section{Other outcome measures}

Recurrent stroke occurred in 14 participants in the intervention group and 11 in the control group

Table $3 \mid$ Comparison of the occurrence of adverse clinical events among participants in the intervention and control groups of the ExStroke Pilot Trial

\begin{tabular}{|c|c|c|c|c|}
\hline \multirow[b]{2}{*}{ Event } & \multicolumn{2}{|c|}{ No (\%) of participants } & \multicolumn{2}{|c|}{ Difference } \\
\hline & Intervention group $(n=157)$ & Control group $(n=157)$ & Hazard ratio $(95 \% \mathrm{Cl})$ & $P$ value \\
\hline Strokes & $14(8.9)$ & $11(7.0)$ & 1.30 (0.59 to 2.87$)$ & 0.51 \\
\hline Myocardial infarction (MI) & $2(1.3)$ & $2(1.3)$ & 1.01 (0.14 to 7.17$)$ & 0.99 \\
\hline Death & $11(7.0)$ & $9(5.7)$ & 1.41 (0.57 to 3.50$)$ & 0.46 \\
\hline Vascular death & $3(1.5)$ & $4(2.5)$ & 0.76 (0.17 to 3.41$)$ & 0.72 \\
\hline Fall fracture (FF) & $5(3.2)$ & $12(7.6)$ & $0.46(0.16$ to 1.31$)$ & 0.14 \\
\hline Stroke, MI, or death & $24(15.3)$ & $19(12.1)$ & 1.37 (0.74 to 2.52 ) & 0.31 \\
\hline Stroke, MI, or vascular death & $18(11.5)$ & $14(8.9)$ & 1.32 (0.66 to 2.65 ) & 0.44 \\
\hline Stroke, MI, death, or FF & $28(17.8)$ & $29(18.5)$ & 1.01 (0.60 to 1.70$)$ & 0.98 \\
\hline First falls & $53(34)$ & $54(34)$ & 1.08 (0.73 to 1.58$)$ & 0.70 \\
\hline
\end{tabular}


Table $4 \mid$ Comparison of the distribution of disability scores among participants in the intervention and control groups of the ExStroke Pilot Trial. Values are numbers (percentages) of participants unless stated otherwise

\begin{tabular}{|c|c|c|c|c|c|c|c|}
\hline \multirow[b]{2}{*}{ Visit } & \multicolumn{6}{|c|}{ Modified Rankin scale score* } & \multirow{2}{*}{$\begin{array}{c}\text { P value of difference } \\
\text { between groups } \dagger\end{array}$} \\
\hline & 0 & 1 & 2 & 3 & 4 & 5 & \\
\hline \multicolumn{8}{|l|}{ Before stroke: } \\
\hline Intervention group & $114(72.6)$ & $28(17.8)$ & $13(8.3)$ & $2(1.3)$ & 0 & 0 & \multirow{2}{*}{ NA } \\
\hline Control group & $116(73.9)$ & $20(12.7)$ & $18(15.5)$ & $3(1.9)$ & 0 & 0 & \\
\hline \multicolumn{8}{|l|}{3 month follow-up: } \\
\hline Intervention group & $23(18.0)$ & $30(23.4)$ & $51(39.8)$ & $17(13.3)$ & $4(3.1)$ & $3(2.3)$ & \multirow{2}{*}{0.10} \\
\hline Control group & $28(20.9)$ & $43(32.1)$ & $43(32.1)$ & $18(13.4)$ & $1(0.7)$ & $1(0.7)$ & \\
\hline \multicolumn{8}{|l|}{6 month follow-up: } \\
\hline Intervention group & $20(16.5)$ & $46(38.0)$ & $38(31.4)$ & $14(11.6)$ & $3(2.5)$ & 0 & \multirow{2}{*}{0.25} \\
\hline Control group & $27(21.4)$ & $50(39.7)$ & $33(26.2)$ & $14(11.1)$ & $2(1.6)$ & 0 & \\
\hline \multicolumn{8}{|l|}{12 month follow-up: } \\
\hline Intervention group & $30(24.8)$ & $37(30.6)$ & $36(29.8)$ & $12(9.9)$ & $4(3.3)$ & $2(1.7)$ & \multirow{2}{*}{0.66} \\
\hline Control group & $28(22.4)$ & $49(39.2)$ & $31(24.8)$ & $11(8.8)$ & $6(4.8)$ & 0 & \\
\hline \multicolumn{8}{|l|}{24 month follow-up: } \\
\hline Intervention group & $32(23.4)$ & $46(33.6)$ & $35(25.5)$ & $15(10.9)$ & $7(5.1)$ & $2(1.5)$ & \multirow{2}{*}{0.88} \\
\hline Control group & $34(23.1)$ & $49(33.3)$ & $38(25.9)$ & $12(8.2)$ & $11(7.5)$ & $3(2.0)$ & \\
\hline
\end{tabular}

*Scale ranges from 0 to 5 , where $0=$ no limitations and $5=$ confinement to bed and requiring constant help.

†Measured by non-parametric Mann-Whitney $U$ test.

(table 3). Participants were readmitted for these events, of which seven were fatal. There were no significant differences regarding the time to recurrent stroke, myocardial infarction, or all cause mortality (table 3). There was no significant difference in disability score (modified Rankin scale) between the two groups at any time point (table 4). Although the qualifying strokes were mild, they did cause an increase in disability from before stroke. At 3 months' follow-up, about 15\%-18\% of participants had a modified Rankin scale of $\geq 3$.

The number of first falls was 53 in the intervention group and 54 in the control group (table 3). Several participants fell more than once; the total number of falls were 93 in the intervention group and 94 in the control group. Falls resulting in fractures were not significantly different in the two groups. The recurrent strokes and fractures contributed to the worsening of modified Rankin scale over time.

\section{DISCUSSION}

Interpretation of results

The main finding of our trial was that repeated encouragement and verbal instruction did not result in a measurable increase in physical activity (in the intention to treat analysis, and in the per protocol analysis among patients who attended all visits). The intervention did not have any significant effect on recurrent vascular events, nor on activity of daily living as measured by the modified Rankin scale.

\section{Strengths and limitations of study}

The strengths of our trial are that it is a multicentre, multinational, randomised clinical trial with masked outcome assessors. The patients had mild strokes and had the physical capability to increase their physical activity. The mixed model analysis is optimal in the presence of missing data (see fig 1) since all observations are used to improve the precision (as compared with a conventional complete patient analysis), and the mixed model analysis will not be biased even if missing PASE scores depend on observed quantities, such as the choice of intervention used. ${ }^{31}$ It was a weakness that we did not test during the trial whether the participants actually increased their physical activity as measured by the PASE score. To confront the patients with the fact that they were not increasing their activity might have given a stronger stimulus to exercise. However, the physiotherapists and other investigators were unaware of the PASE score. Masked assessors, who did not know how the score was calculated, obtained answers to the PASE questionnaire. The repeated questioning about physical activity in the control group may have contributed to a higher PASE score in this group by reminding participants about the importance of physical activity, which would have reduced our chances of finding an intervention effect. We cannot exclude the possibility that some of the PASE assessors became aware of some of the participants' assigned intervention, but this is unlikely to have affected our results.

At the time when this trial was planned, the literature on counselling on physical activity had shown some positive results in elderly sedentary people and in various non-stroke patients. ${ }^{9-11}$ However, systematic reviews from recent years did not find the intervention effective in non-stroke patients. ${ }^{3233}$ Our results in ischaemic stroke confirm the ineffectiveness of counselling on physical activity.

Stroke survivors might be expected to be motivated to improve their level of physical activity. However, that was not apparent in this trial. More than half of the participants' PASE scores derived from household activities, whereas walking outside the home and 


\section{WHAT IS ALREADY KNOWN ON THIS TOPIC}

Observational studies have suggested that physical activity reduces risk of first stroke

In stroke patients there is little evidence on the effect of physical activity on risk of recurrent stroke or other cardiovascular events

Several small trials have shown that supervised group training can result in improved fitness and walking ability in stroke patients over periods of 3 to 6 months

\section{WHAT THIS STUDY ADDS}

This trial of counselling and repeated encouragement to increase physical activity to patients after mild stroke over a period of 24 months did not result in improvement in physical activity

sports activities accounted for only a quarter. In a previous study ${ }^{28}$ a random sample from the general population with similar age had a mean PASE score of about 120 , while patients in this trial had a pre-stroke PASE score that was $35 \%$ to $45 \%$ lower. The lessons to be learnt are that stroke patients are inclined to low levels of physical activity. A contributing reason could be that power in the legs was found to be low bilaterally in a study of muscle power in ambulatory stroke patients. ${ }^{34}$ Verbal encouragement possibly had an effect in some of our participants, but most of this group of patients with mild stroke did not seem to be responsive to our suggestions of change in level of physical activity.

This pilot study was not powered to show an effect of physical training on recurrent stroke, acute myocardial infarction, or survival, which would need more than 10 times the sample size. A Cochrane review ${ }^{35}$ of 12 trials including 289 participants randomised to fitness training versus control concluded that, although it improved walking ability there were too few data for reliable conclusions to be drawn.

\section{Generalisability}

The results of the ExStroke Pilot Trial are probably generalisable to patients with mild ischaemic stroke. Although most of the included participants were Danes, there was no indication that patients from the other countries responded differently. A possible selection bias existed since some patients declined to participate because they were not interested in physical training.

\section{General interpretation}

The best and most cost effective way of increasing physical activity after stroke has not been found.

The aim of our study was to explore the value of a comparatively low cost intervention of repeated verbal encouragement to be physically active. This intervention failed to have the desired effect. Group training has been shown to work over short periods of time. ${ }^{9-13}$ Supervised group training of long duration might be an avenue to be explored. However, it is unknown if increased physical activity will influence risk of recurrent vascular events in stroke survivors. A single recommendation to exercise at the time of discharge from hospital, as it is most often done in stroke units, is unlikely to have any long term effect on behaviour. Our trial results testify to the difficulty of promoting physical activity in stroke patients, who on average had a low PASE score before and after their stroke. However, absence of evidence for an effect is not evidence of absence of an effect. ${ }^{36}$ We therefore need more randomised trials assessing the impact of more compelling interventions designed to improve physical activity among stroke patients.

Details of the ExStroke Pilot Trial Group. Steering committee-G Boysen, T Truelsen, C Gluud. Coordinating investigator and guarantor for the study - G Boysen. Investigators-G Andersen, Anders Christensen, Kim Holsted, L-H Krarup, J Kõrv, Sascha Michelsen, Signe Tellerup Nielsen, Tang Ning, A Oskedra, Mai Roose, Mette Søndergaard, Rikke Thomsen, Sun Xiangrong, Zeng X, Nina Åslund. Study nurse-A Pedersen.

Physiotherapists—Anne Junker Alsted, Krista Ankru, L Hansen, M Lindahl, Monika Pietrzak. Randomisation coordinators and data managementNader Salas, Rita Aslan, Bitten Hansen, Pia Hughes, Lars Klingenberg, C Gluud. Secretary_-Inger Hedegaard. Adjudication committee-Jette Ingerslev, Helle Iversen, Marianne Schroll, Christian Kruse-Larsen, Jesper Sylvest, Bjarne Sigurd, Steen Husted. Independent data monitoring and safety committee-jørgen Hilden, Troels Staehelin Jensen. Statistical analyses-P Winkel.

Funding: The ExStroke Pilot Trial was funded by the Ludvig and Sara Elsass' Foundation, Hede Nielsen Foundation, Eva and Henry Frænkel's Foundation, Søren and Helene Hempel's Foundation, and King Christian $X$ Foundation. The funding sources had no involvement in the design of the trial; data collection, management, analysis, interpretation, and reporting; writing of the paper; or submission of the article for publication. Competing interests: None declared.

1 Lee IM, Hennekens CH, Berger K, Buring JE, Manson JE. Exercise and risk of stroke in male physicians. Stroke 1999;30:1-6.

2 Ellekjær H, Holmen J, Ellekjær L, Vatten L. Physical activity and stroke mortality in women. Ten year follow-up of the Nord-Trøndelag Health Survey. 1984-1986. Stroke 2000;31:14-8.

3 Gillum RF, Mussolino ME, Ingram DD. Physical activity and stroke incidence in women and men: the NHANES I Epidemiologic Followup Study. Am J Epidemiol 1996;143:860- 9.

4 Hu FB, Stampher MJ, Colditz G, Ascherio A, Rexrode KM, Willett WC, Manson JE. Physical activity and risk of stroke in women. JAMA 2000;283:2961-7.

5 Abbott RD, Rodriguez BL, Burchfiel CM, Curb JD. Physical activity in older middle-aged men and reduced risk of stroke: The Honolulu Heart Program. Am J Epidemiol 1994:139:881-93.

6 Lee IM, Paffenbarger RS Jr. Physical activity and stroke incidence: The Harvard Alumni Health Study. Stroke 1998;29:2049-54.

7 Lee CD, Folsom AR, Blair SN. Physical activity and stroke risk: A metaanalysis. Stroke 2003;34:2475-81.

8 Gordon NF, Gulanick M, Costa F, Fletcher G, Franklin BA, Roth EJ, Shephard T. Physical activity and exercise recommendations for stroke survivors: An American Heart Association scientific statement from the council on clinical cardiology, subcommittee on exercise, cardiac rehabilitation, and prevention; the council on cardiovascular nursing; the council on nutrition, physical activity, and metabolism; and the stroke council. Circulation 2004;109:2031-41.

9 Calfas KJ, Long BJ, Sallis JF, Wooten WJ, Pratt M, Patrick K. A controlled trial of physician counseling to promote the adoption of physical activity. Prev Med 1996;25:225-33.

10 Steptoe A, Doherty S, Rink E, Kerry S, Kendrick T, Hilton S. Behavioural counselling in general practice for the promotion of healthy behaviour among adults at increased risk of coronary heart disease:randomised trial. BMJ 1999;319:943-8.

11 Smith BJ, Bauman AE, Bull ML, Booth ML, Harris MF. Promoting physical activity in general practice: a controlled trial of written advice and information materials. Br J Sports Med 2000;34:262-7.

12 Lawlor DA, Hanratty B. The effect of physical activity advice given in routine primary care consultations: a systematic review. J Public Health Med 2001;23:219-26.

13 Eden KB, Orleans CT, Mulrow CD, Pender NJ, Teutsch SM. Does counseling by clinicians improve physical activity? A summary of the evidence for the U.S. preventive Services Task Force. Ann Intern Med 2002;137:208-15.

14 Potempa K, Lopez M, Braun LT, Szidon JP, Fogg L, Tincknell T. Physiological outcomes of aerobic exercise training in hemiparetic stroke patients. Stroke 1995;26:101-5.

15 Duncan P, Richards L, Wallace D, Stoker-Yates J, Pohl P, Luchies C, Ogle A, Studenski S. A randomized, controlled pilot study of a home 
based exercise program for individuals with mild and moderate stroke. Stroke 1998;29:2055-60.

16 Kwakkel G, Wagenaar RC, Twisk JW, Lankhorst GJ, Koetsier JC. Intensity of leg and arm training after primary middle-cerebral-artery stroke: A randomized trial. Lancet 1999;354:191-6.

17 Teixeira-Salmela LF, Nadeau S, McBride I, Olney SJ. Effects of muscle strengthening and physical conditioning training on temporal, kinematic and kinetic variables during gait in chronic stroke survivors. J Rehabil Med 2001;33:53-60.

18 Mead GE, Greig CA, Cunningham I, Lewis SJ, Dinan S, Saunders DH, Phil M, Fitzimons C, Young A. Stroke: A randomized trial of exercise or relaxation. J Am Geriatr Soc 2007;55:892-9.

19 Washburn RA, Smith KW, Jette AM, Janney CA. The Physical Activity Scale for the Elderly (PASE) Development and evaluation. J Clin Epidemiol 1993;46:153-62.

20 Washburn RA, McAuley E, Katula J, Mihalko SL, Boileau RA. The Physical Activity Scale for the Elderly (PASE): Evidence for validity. J Clin Epidemiol 1999;52:643-51.

21 Krarup LH, Gluud C, Truelsen T, Pedersen A, Lindahl M, Hansen L, Michelsen S, Andersen G, Zeng X, Kõrv J, Oskedra A, Boysen G, ExStroke Pilot Trail Group. The ExStroke Pilot Trial: Rationale, design, and baseline data of a randomized multicenter trial comparing physical training versus usual care after an ischaemic stroke. Contemp Clin Trials 2008;29:410-7.

22 Rankin J. Cerebral vascular accidents in patients over the age of 60. II. prognosis. Scott Med / 1957;2:200-15.

23 van Swieten JC, Koudstaal PJ, Visser MC, Schouten HJA, van Gijn J. Interobserver agreement for the assessment of handicap in stroke patients. Stroke 1988;19:604-7.

24 Lindenstrøm E, Boysen G, Christiansen LW, Hansen BR, Nielsen PW. Reliability of Scandinavian neurological stroke scale. Cerebrovasc Dis 1991;1:103-7.

25 Schuit AJ, Schouten EG, Westerterp KR, Saris WH. Validity of the Physical Activity Scale for the Elderly (PASE): according to energy expenditure assessed by the doubly labeled water method. J Clin Epidemiol 1997;50(5):541-6.

26 Martin KA, Rejeski WJ, Miller ME, James MK, Ettinger WH Jr, Messier SP. Validation of the PASE in older adults with knee pain and physical disability. Med Sci Sports Exerc 1999;31(5):627-33.

27 Dinger MK, Oman RF, Taylor EL, Vesely SK, Able J. Stability and convergent validity of the Physical Activity Scale for the Elderly (PASE). J Sports Med Phys Fitness 2004;44(2):186-92.

28 Krarup LH, Truelsen T, Pedersen A, Lerke H, Lindahl M, Hansen L, et al. Level of physical activity in the week preceding an ischemic stroke. Cerebrovascular Diseases 2007;24(2-3):296-300.

29 Lindahl M, Hansen L, Pedersen A, Truelsen T, Boysen G. Self-reported physical activity after ischemic stroke correlates with physical capacity. Advances in Physiotherapy 2008;10:188-94.

30 Rikli RE, Jones Cl. Senior fitness test manual. Champaign, IL: Human Kinetics, 2001:13-50. ISBN: 0736033564

31 Molenberghs G, Kenward MG. Missing data in clinical studies. In: Statistics in practice. J Wiley, 2007.

32 Foster C, Hillsdon M, Thorogood M. Interventions for promoting physical activity. Cochrane Database Syst Rev 2005;(1):CD003180, doi:10.1002/14651858.CD003180.pub2.

33 Hudon C, Fortin $M$, Soubhi H. Single risk factor interventions to promote physical activity among patients with chronic diseases: systematic review. Can Fam Physician 2008;54:1130-7.

34 Saunders DH, Greig CA, Young A, Mead GE. Association of activity limitations and lower-limb explosive extensor power in ambulatory people with stroke. Arch Phys Med Rehabil 2008;89:677-83.

35 Saunders DH, Greig CA, Young A, Mead GE. Physical fitness training for stroke patients. Cochrane Database Syst Rev 2004;(1): CD003316.

36 Gluud LL. Bias in clinical intervention research. Am J Epidemiol 2006;163(6):493-501.

Accepted: 28 April 2009 